\title{
Detection of wind turbines in intertidal areas using SAR polarimetry
}

\author{
Emanuele Ferrentino, Student Member, IEEE, Ferdinando Nunziata, Senior Member, IEEE, \\ Armando Marino, Member, IEEE, Maurizio Migliaccio, Fellow, IEEE, and Xiaoming Li, Member, IEEE
}

\begin{abstract}
The detection of wind turbines in a strong clutter background is analyzed at variance of polarimetric Synthetic Aperture Radar (SAR) configurations. The area of interest is the intertidal zone near Jiangsu, China and two detectors are used, the Polarimetric Notch Filter (PNF) and a change detector that optimizes the ratio between covariance matrices. The detection performance is quantitatively analyzed using the receiver operating characteristic (ROC) curve while the scattering mechanisms that characterize wind turbines are analyzed using the Yamaguchi decomposition. Experimental analysis shows that: a) wind turbines result in a non-trivial scattering mechanism; b) full-polarimetric measurements achieve the best detection performance independently of the two detectors.
\end{abstract}

Index Terms-PolSAR, wind turbines, change detection, notch filter, polarimetry.

\section{INTRODUCTION}

Wind is a sustainable and alternative resource for producing energy and it is one of the green pillars. Wind turbines are widely used at onshore and offshore sites to convert the energy from wind to electrical power [1]. Offshore wind farms have a very low global warming and noise impact. Hereafter, we focus on Rudong County, China, where wind turbines are located in the intertidal flat area together with aquaculture farms. This scenario is very interesting for both scientific and operational viewpoints since it provides an unprecedented opportunity to test the effectiveness and the robustness of multi-polarization Synthetic Aperture Radar (SAR) algorithms to detect metallic targets in a very harsh clutter background. Since wind turbines consist of a metallic tower with blades, polarimetric SAR (PolSAR) detection of wind turbines may be meant similar to the detection of small metallic structures at sea. One key difference relies on the clutter that, in the case of the Rudong County, can be very difficult to be rejected due to the presence of shallow waters, mud flats and aquacultures [2]. This makes the clutter very inhomogeneous and therefore the detection exercise is challenging [3]. To the best of our knowledge, there is only one study that explicitly addressed the detection of wind turbines [4], where a sensitivity analysis is proposed to discuss the enhancement of the target-to-clutter ratio achieved using conventional and compact-polarimetry (CP) PolSAR

E. Ferrentino, F. Nunziata and M. Migliaccio are with the Dipartimento di Ingegneria, Università degli Studi di Napoli Parthenope, Napoli, Italy (e-mail: emanuele.ferrentino@uniparthenope.it; ferdinando.nunziata@uniparthenope.it; maurizio.migliaccio@uniparthenope.it).

A. Marino is with the Department of Natural Sciences, University of Stirling, Stirling, United Kingdom (e-mail: armando.marino@stir.ac.uk).

$\mathrm{X} . \mathrm{Li}$ is with the Institute of Remote Sensing and Digital Earth, Chinese Academy of Sciences, Beijing, China (e-mail: lixm@radi.ac.cn). modes.

Several methods have been proposed in literature to deal with the detection of metallic targets at sea. In [5], convolutional neural networks $(\mathrm{CNN})$ are used for maritime targets detection and classification. Results, obtained by processing TerraSAR-X high-resolution images, demonstrate the benefits of deep-learning in improving detection accuracy. In [6] and [7], a physical dual-polarimetric (DP) approach is proposed to discriminate man-made metallic targets by the sea background according to their different symmetry properties. Experiments, undertaken on TerraSAR-X, RadarSAT-2 and ALOS SAR measurements, show that metallic targets can be distinguished from the surrounding sea. In [8], different sub-look multi-polarimetric detectors are used for vessels detection. Results, undertaken using multi-polarization and multi-frequency SARs, i.e.; RadarSAT-2, TerraSAR-X and ALOS, show the soundness of the proposed approaches. In [9] a detector algorithm based on Polarimetric Notch Filter (PNF) is applied on TerraSAR-X FP SAR data to detect ships. Results show a significant agreement with the available ground truth. Furthermore, the approach resulted to be almost independent of sea conditions. In [10] the sensitivity of CP SAR features to metallic targets at sea is analyzed using CP modes emulated from quad-polarimetric L- and C-band SAR imagery.

This study analyzes the role of polarimetric information to detecting wind turbines at sea in a harsh clutter background. Experiments are carried out considering C- and X-band PolSAR imagery collected by RadarSAT-2 (in full-polarimetric (FP) mode) and TerraSAR-X (in dual-polarimetric (DP) mode) over the Rudong County, Jiangsu Province, see Figure 1 Two PolSAR algorithms are used to observe wind turbines, i.e.; the PNF [9] and a change detector method based on the polarimetric matched filter (PMF) [11][12] since they allow exploiting both FP and DP (VV-HH and VV-VH/HH-HV) and also CP modes [10]. This allows a fair inter-comparison of the detection performance achieved when using different polarimetric channels configurations. To quantify the detection performance the receiver operating characteristic (ROC) is used. The analysis is undertaken at both $\mathrm{C}$ - and $\mathrm{X}$-band. Experimental analysis shows that: a) the scattering mechanism that results from the wind turbines is non-trivial and is characterized by a mixture of polarized (i.e.; single-reflection and double-bouncing) and unpolarized (i.e.; volume component) scattering. In addition, it breaks the reflection symmetry; b) FP measurements result in the best performance since they allow discriminating polarized scattering mechanisms, i.e.; single- 


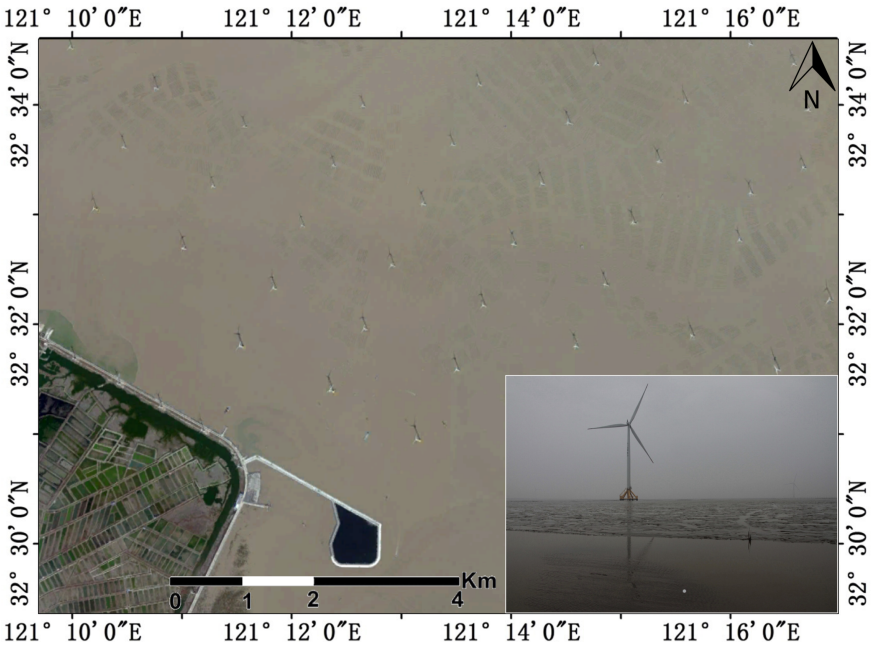

Fig. 1. Google Earth $\left.{ }^{(}\right)$image collected on 12 May, 2014 over the area of Rudong County. On the bottom-right side an enlarged version of the area that includes a wind turbine and the mud flat is shown.

and double bouncing, from the unpolarized backscattering; c) when DP information is available, the VV-HH combination and the $\mathrm{CP}$ modes outperform the VV-VH/HH-HV combinations.

\section{Methodology}

The theoretical rationale that underpins the proposed multipolarization methodologies is here described. First, a brief introduction on the polarimetric observable is given. Then, the two multi-polarization methods are described.

Polarimetric descriptors of complex random scenarios are second-order products of the scattering matrix [13][14]. The positive semi-definite (PSD) and Hermitian covariance matrix is considered:

$$
\mathbf{C}=\left\langle\mathbf{k}_{L} \cdot \mathbf{k}_{L}^{\dagger}\right\rangle
$$

where $\langle\cdot\rangle$ is the finite averaging operator and $\mathbf{k}_{L}$, is the scattering vector, i.e.; the projection of the scattering matrix $\mathbf{S}$ according to the lexicographic basis [13][14] and $\dagger$ means complex conjugate transpose. Since $\mathbf{C}$ is a PSD Hermitian matrix, it can be uniquely decomposed according to the eigenvalue/eigenvector decomposition [15]:

$$
\mathbf{C}=\mathbf{U D U}^{T}
$$

where $\mathbf{U}$ and $\mathbf{U}^{T}$ are unitary orthogonal matrices and $\mathbf{D}$ is a diagonal matrix whose elements are the real and non negative eigenvalues of $\mathbf{C}$.

When dealing with turbines, we expect a complex polarimetric signature which consists of pixels characterized by a dominant deterministic mechanism (i.e.; single reflection, double-bouncing, etc.) and pixels resulting in depolarization [16]. Hence, a robust detector should be not tuned on the discrimination of a single specific scattering mechanism that characterizes the target, indeed it should be able to include all the scattering mechanisms that distinguish the target from the background clutter. Hence, we selected two detectors that are described in the following subsections.

\section{A. PNF method}

This method exploits the polarimetric coherence to enhance differences between targets and the clutter background: targets are considered as anomalies with respect to the scattering of the background.

Hence, the polarimetric coherence between the sea clutter and its perturbed version can be defined as follows [9]:

$$
\gamma=\frac{1}{\sqrt{1+\operatorname{Red} R \frac{P_{\text {sea }}}{P_{T}}}}
$$

where the parameter RedR is a tuning parameter that allows rescaling the clutter distribution to avoid numerical errors in the computation of the detection mask [9]. Note, however, that the theoretical detection performance is independent of RedR [9]. $P_{\text {sea }}$ and $P_{T}$ stand for the power of the sea surface clutter and the target, respectively. The total power related to the observed scene is defined as:

$$
P_{\text {tot }}=\mathbf{t}^{\dagger} \mathbf{t}
$$

where $\mathbf{t}$ is the feature partial scattering vector obtained projecting $\mathbf{C}$ onto a lexicographic basis. This power can be decomposed into a term associated to the sea surface, $P_{\text {sea }}$, and a term that includes "non-sea" targets. The sea clutter power is given by:

$$
P_{\text {sea }}=\left|\mathbf{t}^{\dagger} \mathbf{t}_{\text {sea }}\right|^{2}
$$

where $\mathbf{t}_{\text {sea }}$ is the partial target extracted from a sea clutter reference area. Note that, the square operator is necessary because $\mathbf{t}_{\text {sea }}$ is a unitary vector. The power of the "non-sea" targets is given by:

$$
P_{T}=P_{t o t}-P_{\text {sea }}
$$

In this study, $\gamma(3)$ is exploited to observe wind turbines.

\section{B. Change detection method}

The method is based on the PMF that, proposed in the seminal study of Novak et al. [11] to deal with the optimization of the target-to-clutter power ratio using a single polarimetric SAR image, has been extended in [17] to deal with the optimization of the power ratio between two different polarimetric SAR acquisitions. Hence, the method used in this study to observe wind turbines against the clutter is based on the scattering mechanisms $\underline{\omega}_{\max }$ that maximize the following ratio:

$$
\rho_{12}=\frac{\underline{\omega}^{\dagger} \mathbf{C}_{11} \underline{\omega}}{\underline{\omega}^{\dagger} \mathbf{C}_{22} \underline{\omega}}
$$

where $\mathbf{C}_{22}$ and $\mathbf{C}_{11}$ are covariance matrices that describe the reference scenario (i.e.; the clean sea surface) and the test area, respectively, and $\underline{\omega}$ is the projecting vector. Both covariance matrices, in this study, are estimated using a $3 \times 3$ boxcar 
window. To maximize (7), an optimization problem is to be solved. In this study, the Lagrangian method [12][13][14][17] is applied to the quadratic form $\underline{\omega}^{\dagger} \mathbf{C}_{\mathrm{CD}} \underline{\omega}$ where the change matrix $\mathbf{C}_{C D}$ is given by

$$
\mathbf{C}_{C D}=\mathbf{C}_{11} \mathbf{C}_{22}^{-1}
$$

Hence, the following eigenvalue problem is obtained [11]:

$$
\mathbf{C}_{C D \underline{\omega}}=\lambda_{i} \underline{\omega}
$$

with $\lambda_{i}$ (with $i=1,2,3$ ) being the real eigenvalues that maximize/minimize the ratio between the covariance matrices associated with the test area and the reference scenario, respectively.

In this study, the sum of the absolute value of the three eigenvalues is considered to detect anomalies with respect to the clean sea surface:

$$
\lambda=\left|\lambda_{1}\right|+\left|\lambda_{2}\right|+\left|\lambda_{3}\right|
$$

$\lambda$ is expected to be sensitive to the peculiar scattering mechanisms that make the polarimetric signature of the metallic turbines different from the clutter. Note that when DP PolSAR data are available, eq. (10) consists of only two eigenvalues; hence, only partial information about the scattering scene can be obtained.

In this study, $\lambda$ is exploited to observe wind turbines.

\section{EXPERIMENTS}

In this section, showcases related to C-band RadarSAT2 FP and X-band TerraSAR-X DP HH/VV SAR data (see Table I], are discussed. Wind conditions, estimated from the SAR imagery according to [18] and [19], belong to the lowto-moderate regime, see Table [1] The Jiangsu coast is mainly characterized by mud flats that are well visible in the optical image in Figure 1. A large number of wind turbines is located in the Jiangsu intertidal zone, see the white dots in the image of Figure 1. In the bottom right part of Figure 1, a camera picture of the mud flat and wind turbine is shown.

To discuss the added-value information provided by polarimetric measurements to distinguish wind turbines from both the sea surface and the more challenging mud flat environment, the two detectors are exploited using different combinations of polarimetric channels. The quantitative analysis is undertaken using the ROC curve evaluated on the $\gamma$ and $\lambda$ imagery. The ROC consists of plotting the probability of false alarm $P_{f a}$ with respect to the probability of detection $P_{d}$ by varying the threshold between a minimum and a maximum value that depends on the histogram of $\gamma$ and $\lambda$ [8]. In particular, $P_{d}$ is given by the ratio of the detected wind turbines over the total number of wind turbines visually inspected in the SAR imagery. Visual inspection is undertaken geocoding the SPAN image and superimposing it on an optical image. To evaluate $P_{f a}$, an area (see Figure 2 (a) and (b)), that includes both aquacultures and intertidal flat but does not contain land or evident ambiguities, is selected. Then, the signatures of wind
TABLE I

SAR DATASETS ACQUIRED BY RADARSAT-2 AND TERRASAR-X

\begin{tabular}{cccccccc}
\hline \hline Dataset & Sensor & Polarization & $\begin{array}{c}\text { Acquisition } \\
\text { date }\end{array}$ & Band & $\begin{array}{c}\text { Angle of } \\
\text { incidence } \\
(\text { AoI }) \\
\left({ }^{\circ}\right)\end{array}$ & $\begin{array}{c}\text { Wind } \\
\text { speed } \\
(\mathbf{m} / \mathbf{s})\end{array}$ & $\begin{array}{c}\text { Number } \\
\text { of wind } \\
\text { turbines }\end{array}$ \\
\hline \hline 1 & & & & & & \\
2 & RS-2 & FP & $10 / 04 / 2014(9: 50: 53)$ & C & 29 & 5.49 & 62 \\
3 & TS-2 & FP & $17 / 04 / 2014(9: 46: 44)$ & C & 22 & 4.70 & 88 \\
4 & TS-X & DP (HH-VV) & $10 / 04 / 2014(9: 52: 54)$ & X & 40 & 5.49 & 78 \\
\hline \hline
\end{tabular}

turbines are manually masked out and the $P_{f a}$ is given by the ratio between the number of detected pixels over the total number of pixels.

The first experiment deals with the RadarSAT-2 scenes acquired on 10 April 2014 (see dataset 1 in Table I) and 17 April 2014 (see dataset 2 in Table \). An excerpt of the HHpolarized squared modulus SAR data related to dataset 1 is shown in graytones $(\mathrm{dB})$ in Figure $2(a)$, while the output of the PNF and the PMF are shown in false color in Figure 2 (c) and (e), respectively. Both detectors correctly emphasize wind turbines with respect to the sea background and, more interestingly, wind turbines are also well-distinguishable from the intertidal flat. The output of the PMF looks noisier than the PNF one, witnessing that this method exhibits a pronounced sensitivity to even small changes between the scattering mechanisms of the reference area and the observed scene. On the other side, PNF being a notch filter, is better tuned on the detection of departures from the quasi-deterministic Bragg scattering. To better analyze the added-value of polarimetric information to detect wind turbines, the ROC curve related to the dataset 1 is evaluated using both FP and DP polarimetric combinations and considering the single-polarization $\mathrm{HH}$ and HV channels as benchmark, see Figure 3 Note that, in the single-polarization case, the ROC curve is evaluated using the $5 \times 5$ speckle-filtered $\mathrm{HH}$ and $\mathrm{HV}$ intensity image adopting the same procedure followed in the polarimetric case. The $\operatorname{RedR}$ parameter, after a cut-and-try process, is fixed to 0.7 since ensuring the best trade-off. In this case, 62 wind turbines are used as test. We note that polarimetric measurements, together with appropriate polarimetric filters, are beneficial to achieve better results with FP resulting in the best performance. The comparison of the PNF and PMF shows that the latter performs slightly better, for a given polarimetric combination. To provide quantitative results, a figure of merit is introduced that consists of evaluating the area between the ROC curves and the left top part of the plot, see Table III. The area is calculated using a rectangle whose right-most side is bounded by the ROC curve resulting in the lowest $P_{f a}$ value, see Figure 3 The detector performance improves as the area gets smaller. In Table $[1$ results relevant to emulated $\mathrm{CP}$ modes are also listed [20]. In particular, linear +45 and right-hand circular (RHC) CP modes are considered [20]. The figure of merit shows that FP information always guarantees the best performance, independently of the two detectors. Hence, FP performance is considered as benchmark to quantify the performance loss (PL) (listed in percentage in Table III) when partial polarimetric data are used. The PL are always within $46 \%$; hence, all the PolSAR modes perform better than the single-polarization ones. The performance of $\mathrm{CP}$ and $\mathrm{HH}-\mathrm{VV}$ 
TABLE II

FIGURE-OF-MERIT: AREA BETWEEN THE ROC CURVES AND THE LEFT TOP PART AND PERFORMANCE LOSS (PL) IN BRACKETS.

\begin{tabular}{ccccc}
\hline \hline & Dataset 1 & Dataset 2 & Dataset 3 & Dataset 4 \\
\hline \hline$\gamma_{F P}$ & $6.01 \mathrm{e}-05$ & $8.14 \mathrm{e}-05$ & - & - \\
$\gamma_{H H-V V}$ & $6.93 \mathrm{e}-05(15 \%)$ & $8.96 \mathrm{e}-05(10 \%)$ & $2.07 \mathrm{e}-05$ & $1.96 \mathrm{e}-05$ \\
$\gamma_{V V-V H}$ & $8.11 \mathrm{e}-05(34 \%)$ & $1.19 \mathrm{e}-04(46 \%)$ & - & - \\
$\gamma_{H} H-H V$ & $8.46 \mathrm{e}-05(40 \%)$ & $1.19 \mathrm{e}-04(46 \%)$ & - & - \\
$\gamma$ CP linear+45 & $6.79 \mathrm{e}-05(12 \%)$ & $8.87 \mathrm{e}-05(9 \%)$ & - & - \\
$\gamma$ CP RHC & $6.62 \mathrm{e}-05(10 \%)$ & $8.79 \mathrm{e}-05(8 \%)$ & - & - \\
\hline$\lambda_{F P}$ & $5.45 \mathrm{e}-05$ & $7.99 \mathrm{e}-05$ & - & - \\
$\lambda_{H H} H V$ & $6.37 \mathrm{e}-05(17 \%)$ & $9.05 \mathrm{e}-05(13 \%)$ & $1.99 \mathrm{e}-05$ & $1.84 \mathrm{e}-05$ \\
$\lambda_{V V-V H}$ & $7.53 \mathrm{e}-05(38 \%)$ & $1.09 \mathrm{e}-04(36 \%)$ & - & - \\
$\lambda_{H} H-H V$ & $7.95 \mathrm{e}-05(46 \%)$ & $1.15 \mathrm{e}-04(44 \%)$ & - & - \\
$\lambda$ CP linear+45 & $6.31 \mathrm{e}-05(16 \%)$ & $8.94 \mathrm{e}-05(12 \%)$ & - & - \\
$\lambda$ CP RHC & $6.53 \mathrm{e}-05(20 \%)$ & $1.03 \mathrm{e}-04(30 \%)$ & - & - \\
\hline$\sigma_{0}^{H H}$ & $1.92 \mathrm{e}-04$ & $1.80 \mathrm{e}-04$ & $7.06 \mathrm{e}-05$ & $7.45 \mathrm{e}-05$ \\
$\sigma_{0}^{H V}$ & $1.43 \mathrm{e}-04$ & $1.78 \mathrm{e}-04$ & - & - \\
$\sigma_{0}^{V} V$ & $1.97 \mathrm{e}-04$ & $1.89 \mathrm{e}-04$ & $7.13 \mathrm{e}-05$ & $7.75 \mathrm{e}-05$ \\
\hline \hline
\end{tabular}

modes is very close to the FP mode (i.e.; within 20\%); while HH-VV / VV-VH combinations provide the worst result (i.e.; within 46\%).

These results can be physically explained by analyzing the scattering-based information carried on the polarimetric channels combinations. The model-based decomposition, proposed by Yamaguchi et al. [21], is applied to analyze the dominant scattering mechanisms. Results show that the mean scattering mechanism consists of $21 \%$ of surface component, $24 \%$ of helix component, $27 \%$ of double-bouncing and $28 \%$ of volume component. Note that these percentages are obtained by averaging values obtained for all the visually inspected turbines. Hence, wind turbines are characterized by a nontrivial scattering mechanism that is not dominated by doublebouncing since it includes both a polarized component (i.e.; surface and double bouncing), and a depolarizing one (i.e.; volume scattering). In addition, wind turbines, being manmade metallic targets, broke the reflection symmetry property that characterizes natural target [7] leading to a non-negligible helix component. This is in contrast to what naively expected. The detection performance of the polarimetric modes can be explained considering that: a) full-polarimetric measurements show sensitivity to all the above-mentioned mechanisms; b) partial polarimetric modes show sensitivity to a subset of these mechanisms. In particular, DP HH-VV and $\mathrm{CP}$ modes result in a sensitivity closer to FP one; while the co-cross combinations are mostly sensitive to the departure from the reflection symmetry. The ROC curves related to the dataset 2 (not shown to save space) agree with the previous ones, as well as the figure of merit listed in Table II. Note that, in this case, 88 wind turbines samples are considered.

The second experiment deals with the TerraSAR-X scenes acquired on 10 April 2014 (dataset 3) and 16 April 2014 (dataset 4), see Table II. An excerpt of the HH-polarized squared modulus SAR data related to dataset 3 is shown in graytones $(\mathrm{dB})$ in Figure $2(b)$; while the output of the PNF and the PMF are shown in false colors in Figures 2 (d) and $(f)$, respectively. Even in this case, both detectors correctly emphasize wind turbines with respect to the clutter background. The ROC curves evaluated using the different polarimetric combinations are shown in Figure 4 where the

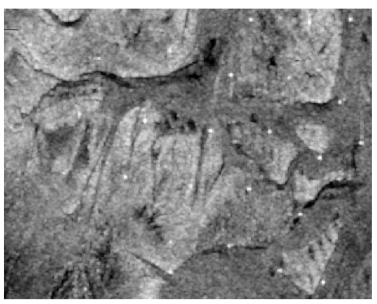

(a)

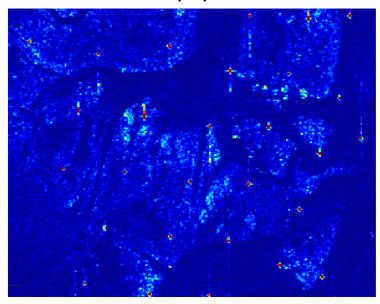

(c)

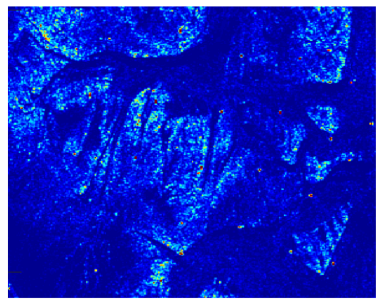

(e)

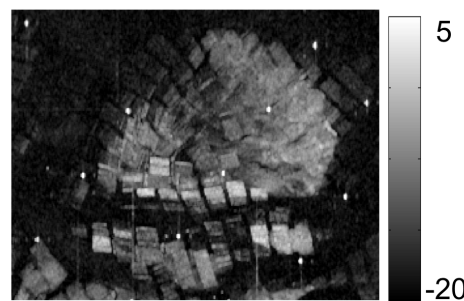

(b)

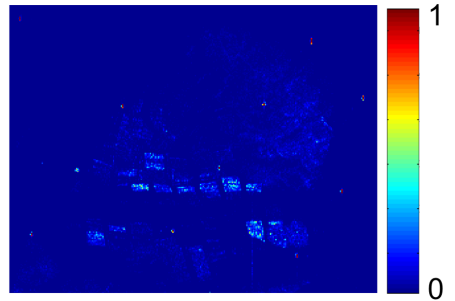

$(d)$

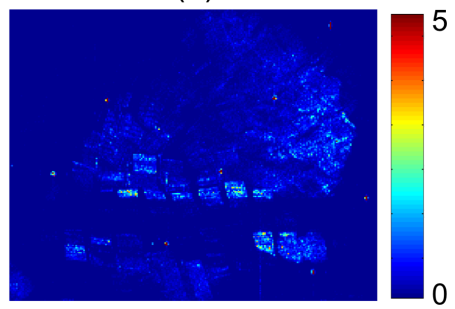

$(f)$
Fig. 2. Excerpts of SAR scenes collected by RadarSAT-2 (first column) and TerraSAR-X (second column) both on 10 April 2017. Rows refer to the outputs of the PNF (second row) and change detector (third row); while the first row shows $\mathrm{HH}$-polarized grayscale image in $\mathrm{dB}$.

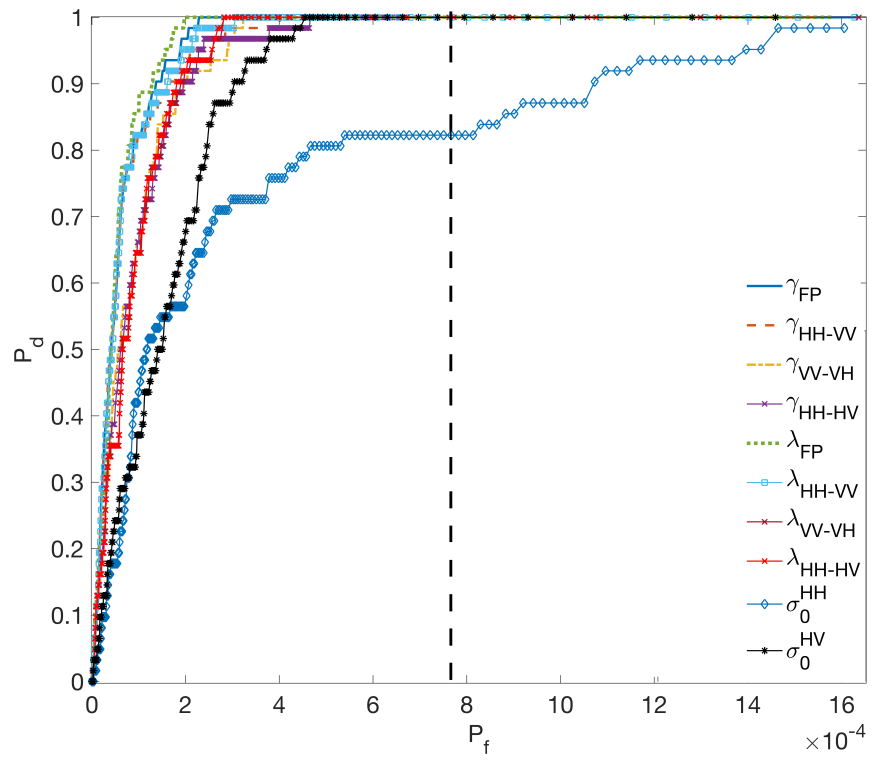

Fig. 3. ROC curve related to the RadarSAT-2 dataset acquired on 10/04/2014.

speckle filtered single-polarization $\mathrm{HH}$ and VV intensity channels are also shown for reference purposes. In this case, the number of wind turbines is equal to 78. Qualitative results are similar to what formerly experienced in the RadarSAT2 cases. The quantitative results provided by the figure of 


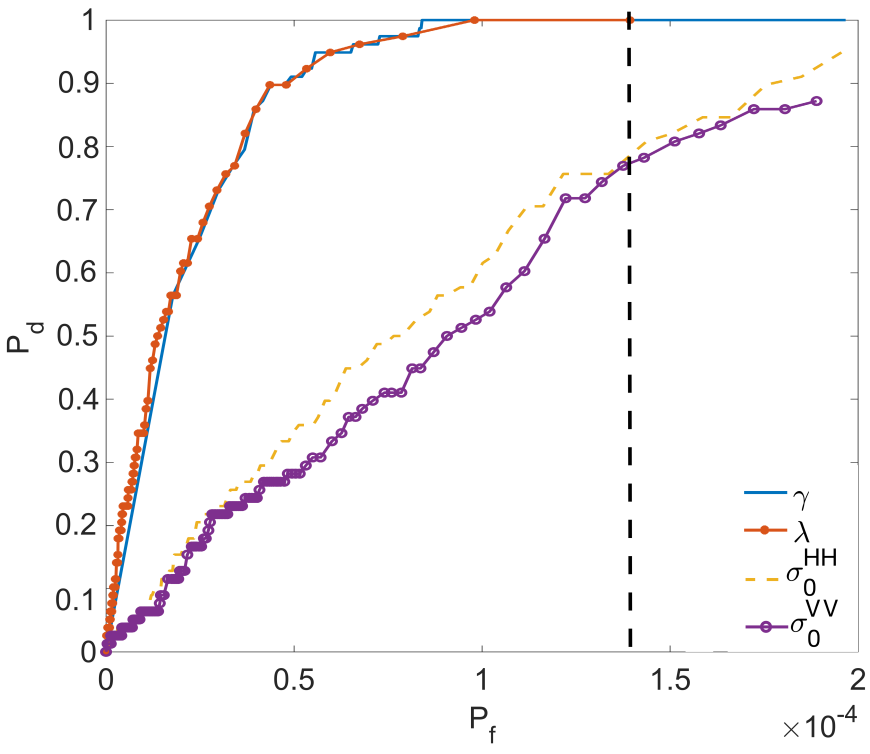

Fig. 4. ROC curve related to the TerraSAR-X dataset acquired on 10/04/2014.

merit, see Table $\Pi$. confirm the visual inspection of the ROC curves, demonstrating that the best performance is achieved by the polarimetric mode that results in the highest information content (in this case DP). The ROC curves related to dataset 4 , where 53 wind turbines are considered, agree with the previous ones (not shown to save space).

\section{CONCLUSions}

In this study, the detection of wind turbines in a strong clutter environment is analyzed at variance of polarimetric SAR configurations using actual data sets acquired by the C-band RadarSAT-2 and the X-band TerraSAR-X sensors. Two detectors are used and the performance is analyzed at variance of polarimetric configurations using ROC curves. Experimental results show that FP measurements always guarantee the best performance independently by the polarimetric detector choice. In addition, wind turbines result in a nontrivial polarimetric scattering behavior that, in contrast to what naively expected, cannot be simply described by a dominant double-bounching mechanism.

\section{ACKNOWLEdgments}

This study is partly funded by the European Space Agency (ESA) in the framework of the Dragon 4 cooperation between ESA and Chinese Ministry of Science and Technology, project ID 32235, and by the Università degli Studi di Napoli Parthenope, "Fondi di ricerca individuale".

\section{REFERENCES}

[1] X. Li and S. Lehner, "Observation of TerraSAR-X for studies on offshore wind turbine wake in near and far fields," IEEE J. Sel. Topics Appl. Earth Observ. Remote Sens., vol. 6, no. 3, pp. 1757-1768, June 2013.

[2] X. Geng, X.-M. Li, D. Velotto, and K.-S. Chen, "Study of the polarimetric characteristics of mud flats in an intertidal zone using C- and X-band spaceborne SAR data," Remote Sens Environ, vol. 176, pp. 56 $-68,2016$.
[3] J.-S. Lee and I. Jurkevich, "Coastline detection and tracing in SAR images," IEEE Trans. Geosci. Remote Sens., vol. 28, pp. 662-668, jul 1990.

[4] H. Li, W. Perrie, Y. He, S. Lehner, and S. Brusch, "Target detection on the ocean with the relative phase of compact polarimetry SAR," IEEE Trans. Geosci. Remote Sens., vol. 51, no. 6, pp. 3299-3305, June 2013.

[5] C. Bentes, D. Velotto, and B. Tings, "Ship classification in TerraSAR$\mathrm{X}$ images with convolutional neural networks," IEEE J. Ocean. Eng., vol. 43, no. 1, pp. 258-266, Jan 2018.

[6] D. Velotto, F. Nunziata, M. Migliaccio, and S. Lehner, "Dualpolarimetric TerraSAR-X SAR data for target at sea observation," IEEE Geosci. Remote Sens. Lett., vol. 10, no. 5, pp. 1114-1118, March 2013.

[7] F. Nunziata, M. Migliaccio, and C. E. Brown, "Reflection symmetry for polarimetric observation of man-made metallic targets at sea," IEEE $J$. Ocean. Eng, vol. 37, no. 3, pp. 384-394, July 2012.

[8] A. Marino, M. J. Sanjuan-Ferrer, I. Hajnsek, and K. Ouchi, "Ship detection with spectral analysis of synthetic aperture radar: A comparison of new and well-known algorithms," Remote Sens., vol. 7, no. 5, pp. 5416-5439, April 2015

[9] A. Marino, "A notch filter for ship detection with polarimetric SAR data," IEEE J. Sel. Topics Appl. Earth Observ. Remote Sens., vol. 6, no. 3, pp. 1219-1232, June 2013.

[10] R. L. Paes, F. Nunziata, and M. Migliaccio, "On the capability of hybridpolarity features to observe metallic targets at sea," IEEE J. Ocean. Eng., vol. 41, no. 2, pp. 346-361, April 2016.

[11] L. M. Novak, M. B. Sechtin, and M. J. Cardullo, "Studies of target detection algorithms that use polarimetric radar data," IEEE Trans. Aerosp. Electron. Syst., vol. 25, no. 2, pp. 150-165, March 1989.

[12] A. Marino and A. Alonso-Gonzalez, "An optimization of the difference of covariance matrices for PolSAR change detection," in 2017 IEEE International Geoscience and Remote Sensing Symposium (IGARSS), July 2017.

[13] S. Cloude, Polarisation: Applications in Remote Sensing. OUP Oxford, 2009.

[14] J. Lee and E. Pottier, Polarimetric Radar Imaging: From Basics to Applications. CRC Press, 2009.

[15] S. R. Cloude and E. Pottier, "An entropy based classification scheme for land applications of polarimetric SAR," IEEE Trans. Geosci. Remote Sens., vol. 35, no. 1, pp. 68-78, Jan 1997.

[16] A. Marino, D. Velotto, and F. Nunziata, "Offshore metallic platforms observation using dual-polarimetric TS-X/TD-X satellite imagery: A case study in the Gulf of Mexico," IEEE J. Sel. Topics Appl. Earth Observ. Remote Sens., vol. 10, no. 10, pp. 4376-4386, Oct 2017.

[17] A. Marino and I. Hajnsek, "A change detector based on an optimization with polarimetric SAR imagery," IEEE Trans. Geosci. Remote Sens., vol. 52, no. 8, pp. 4781-4798, Aug 2014.

[18] V. Corcione, G. Grieco, M. Portabella, F. Nunziata, and M. Migliaccio, "A novel azimuth cut-off implementation to retrieve sea surface wind speed from SAR imagery," IEEE Trans. on Geosci. and Remote Sens., In print 2018

[19] G. Benassai, D. D. Luccio, V. Corcione, F. Nunziata, and M. Migliaccio, "Marine spatial planning using high-resolution synthetic aperture radar measurements," IEEE J. Oceanic Eng., vol. 43, no. 3, pp. 586-594, July 2018.

[20] A. Buono, F. Nunziata, and M. Migliaccio, "Analysis of full and compact polarimetric SAR features over the sea surface," IEEE Geosci. Remote Sens. Lett., vol. 13, no. 10, pp. 1527-1531, Oct 2016.

[21] Y. Yamaguchi, T. Moriyama, M. Ishido, and H. Yamada, "Fourcomponent scattering model for polarimetric SAR image decomposition," IEEE Trans. on Geosci. and Remote Sens., vol. 43, no. 8, pp. 1699-1706, Aug 2005. 